\title{
Neural stem cell transplantation combined with erythropoietin for the treatment of spinal cord injury in rats
}

\author{
YAN ZHAO $^{1 *}$, YUAN ZUO $^{1 *}$, JIANMING JIANG $^{2}$, HUIBO YAN $^{3}$, XILIANG WANG ${ }^{1}$, \\ HUNJUN HUO $^{1}$ and YULONG XIAO ${ }^{1}$

\begin{abstract}
${ }^{1}$ Department of Thoracolumbar Spine Surgery, The Second Affiliated Hospital of Inner Mongolia Medical University, Hohhot, Inner Mongolia 010030; ${ }^{2}$ Department of Orthopedic and Spinal Surgery, Nanfang Hospital, Southern Medical University, Guangzhou, Guangdong 510515; ${ }^{3}$ Department of Spinal Surgery, Third Affiliated Hospital, Southern Medical University, Guangzhou, Guangdong 510630, P.R. China
\end{abstract}

Received September 12, 2014; Accepted June 8, 2015

DOI: $10.3892 /$ etm.2016.3677

\begin{abstract}
Spinal cord injury (SCI) comprises nerve and motor function disorders that may be caused by a variety of damaging factors and is challenging to treat. The aim of the present study was to investigate the regenerative effects of neural stem cell (NSC) transplantation combined with intraperitoneal injection of erythropoietin (EPO) on cross-sectional SCI in rats. A model of SCI was induced in 40 adult Wistar rats via the complete transection of the 10th thoracic vertebra (T10). The rats were allocated at random into 4 groups: Control, NSC, $\mathrm{EPO}$ and NSC + EPO groups (n=10 per group). Morphological alterations associated with axonal regeneration were detected using neurofilament (NF)-200 immunohistochemistry and immunofluorescence staining after 8 weeks. Basso, Beattie and Bresnahan (BBB) scoring was used to evaluate the recovery of hindlimb function. A total of 5 rats died following surgery, including 2 control rats and 1 rat each in the EPO, NSC and NSC + EPO groups. NSCs labeled with bromodeoxyuridine were observed to have survived and migrated in the spinal cord tissue after 8 weeks. Significant histomorphological differences were observed in the NSC and NSC + EPO groups compared with the EPO and control groups. Furthermore, the rats of the NSC + EPO group exhibited significantly enhanced axonal regeneration in the SCI area compared with the NSC group rats. The rats of the NSC and NSC + EPO groups exhibited significantly improved BBB scores compared with the EPO and control group rats at 7 days after treatment $(\mathrm{P}<0.05)$.
\end{abstract}

Correspondence to: Professor Jianming Jiang, Department of Orthopedic and Spinal Surgery, Nanfang Hospital, Southern Medical University, 1838 Guangzhou Dadao Bei, Guangzhou, Guangdong 510515, P.R. China

E-mail: jiangjianminggz@qq.com

\section{*Contributed equally}

Key words: neural stem cells, erythropoietin, cell transplantation, spinal cord injury
In addition, the BBB scores of the NSC + EPO group were significantly improved compared with those of the three other groups at 7 days after surgery $(\mathrm{P}<0.05)$. Therefore, the results of the present study suggest that NSC transplantation combined with intraperitoneal injection of EPO may benefit the survival and regeneration of injured axons, and accelerate the repair of injured spinal cord tissue, thus facilitating the functional recovery of hindlimb locomotor function in rats.

\section{Introduction}

Spinal cord injury (SCI) is a nerve and motor function disorder that may be caused by a variety of damaging factors. SCI is a serious health challenge worldwide, which has resulted in the investigation of a range of novel technologies and methods for the potential treatment of SCI.

A previous study observed that neural stem cells (NSCs) exhibit a number of advantages compared with other types of stem cells in a rat model of stroke, including enhanced proliferation and self-renewal potential, facilitating the induction and operation of NSCs in vitro, early embryonic cell characteristics and the capacity to differentiate into various types of nervous system cell (1). NSCs are a type of stem cell with multiple differentiation potential, which are able to proliferate and self-renew, differentiate into astrocytes, neurons and oligodendrocytes. Therefore, NSCs are ideal seed cells for transplantation. In the present study, tNSCs extracted from fetal rat hippocampus were cultured in vitro, which significantly increases the number, activity and purity of the primary NSCs. Thus, NSCs may be a more effective stem cell type for the treatment of SCI. However, the simple application of neural stem cells for treatment of spinal cord injury is not ideal. It was previously considered that EPO is only a endocrine hormone acting on hematopoietic cells. However, EPO has been demonstrated to be a type of tissue protection factor, which exerts neurotrophic and neuroprotective effects $(2,3)$. Yuan et al (4) observed the effects of erythropoietin on the differentiation of embryonic cerebral cortex neural stem cells of rats cultured in vitro, the results indicated that erythropoietin is able to promote the differentiation of neural stem cells into neurons. Therefore, the aim of the present study was to investigate the 
effectiveness of a combination of NSC transplantation and EPO administration for the treatment of SCI in rats, and to compare the combined treatment with NSC transplantation alone in order to elucidate possible underlying mechanisms.

\section{Materials and methods}

Animal sources. A study population of 40 healthy adult female Wistar rats, weighing $220 \pm 20 \mathrm{~g}$, in addition to 2 Wistar rats that were 14 days pregnant, were provided by the Laboratory Animal Center of Inner Mongolia University (Hohhot, China). This study was approved by the ethics committee of Inner Mongolia Medical University.

Reagents and instruments. Dulbecco's modified Eagle's medium (DMEM)/F12 media, fetal bovine serum (FBS) and B27 supplements were purchased from Gibco Life Technologies (Beijing, China). EPO was obtained from Shenyang Sunshine Pharmaceutical Co., Ltd., (Shenyang, China), while basic fibroblast grow th factor (bFGF) and epidermal growth factor (EGF) were purchased from Sigma-Aldrich (St. Louis, MO, USA). A StreptAvidin-Biotin Complex (SABC) immunohistochemical kit, polyclonal rabbit anti-nestin (AB5922; EMD Millipore, Billerica, MA, USA), mouse anti-bromodeoxyuridine (BrdU; ANT-049) and anti-neurofilament (NF)-200 (BYK-0708R; Boyanbio, Shanghai, China) antibodies were used. Fluorescein isothiocyanate (FITC), trypsin and $25-\mathrm{cm}^{2}$ culture bottles were obtained from Corning Incorporated (Corning, New York, NY, USA), a surgical instrument and operating microscope (SXP1C; Shanghai Medical Optical Instrument Co., Ltd., Shanghai, China). A set of surgical instruments and devices including a fiber operation knife, scissors, tweezers and forceps were also obtained from this company.

Primary culture and identification of NSCs. Experimental methods were based on those described in a previous study (5). The pregnant rats were decapitated while anesthetized with a $3.5 \%$ intraperitoneal injection of pentobarbital $(1.3 \mathrm{ml} / 100 \mathrm{~g})$, and the fetal rats were immediately removed via caesarian section under sterile conditions. An incision was made in the skull in order to obtain the brain tissue, and tissues of the midbrain, hippocampus and subventricular zones were separated. Isolated fetal rat brain tissue was immersed in D-Hanks solution, then $1-\mathrm{mm}^{3}$ tissue sections were obtained using ophthalmic scissors, digested with $2 \mathrm{ml}$ of $0.125 \%$ trypsin at $37^{\circ} \mathrm{C}$ for $10-15 \mathrm{~min}$ and subjected to repeated mechanical pipetting. Next, $4 \mathrm{ml}$ of 10\% FBS in DMEM/F12 was added to terminate the digestion. The mixture was filtered through a 200-mesh stainless steel screen and centrifuged for $10 \mathrm{~min}$ at $125.6 \mathrm{x}$ g. The supernatant was discarded and complete medium consisting of DMEM/F12 + B27 (2 ml/100 ml), bFGF $(10 \mathrm{ng} / \mathrm{ml})$ and EGF $(20 \mathrm{ng} / \mathrm{ml})$ was added. A single cell suspension was administered to flasks at a living cell concentration of $5-8$ cells $/ \mathrm{ml}$. The cell suspension was cultured in a $\mathrm{CO}_{2}$ incubator for 5-7 days, centrifuged for $5 \mathrm{~min}$ at $125.6 \mathrm{x} \mathrm{g}$ and half the quantity of the medium was changed every 2-3 days. The NSCs at passages 3 or 4 were used to create a cell suspension, and the cell concentration was adjusted to $1 \times 10^{5}$ cells/ $\mu 1(6,7)$. Subsequently, immunohistochemical and immunofluorescence analysis of the nestin antigen, a neural stem cell marker, was conducted at the Laboratory of Southern Medical University (Guangzhou, China).

Detection of BrdU-labeled NSCs. Experimental methods are based on those described in a previous study (8). An extracted fraction of the single cell suspension was inoculated into a culture flask and combined with BrdU solution at a concentration of $10 \mu \mathrm{mol} / 1$ in complete DMEM/F12 medium. The cell suspension was prepared after incubation for $48 \mathrm{~h}$, and the cell concentration was adjusted to $1 \times 10^{5}$ cells/ $\mu 1$ for alternation. The expression of nestin antigen was then evaluated using immunohistochemistry and immunofluorescence analysis.

Establishing the completely transected spinal cord model of SCI. The completely transected spinal cord model was established in rats according to a previously described protocol (9). Rats were anesthetized using 3.5\% pentobarbital $(1.3 \mathrm{ml} / 100 \mathrm{~g})$ via intraperitoneal injection, and then the T10 spinous process segments were exposed under sterile conditions (10). Completely lifted the spinal cord with the blunt crochet, the T10 segment was cut in addition to the dura and spinal cord tissue using a scalpel at two positions to isolate a 1-mm spinal segment. The transected spinal cord tissue was then removed using bent-ended tweezers, and a gelatin sponge was used to fill the space in the injured spinal cord (Fig. 1).

Grouping of the experimental animals. A total of 40 adult Wistar rats were allocated at random into four groups, namely the NSC, NSC + EPO, EPO and control groups ( $\mathrm{n}=10$ per group). In the NSC group, following modeling, the NSC suspension was centrifuged for $5 \mathrm{~min}(157 \mathrm{x}$ g) using a benchtop centrifuge (TD4A-WS; Changsha Weierkang Xiangying Centrifuge Co., Ltd., Changsha, China) and the cell concentration was adjusted to $1 \times 10^{5}$ cells/ $\mu 1$ with DMEM. Next, $10 \mu 1$ NSC suspension was injected into a gelatin sponge and in the places of $1 \mathrm{~mm}$ at the top and bottom of each lesion using a micro-syringe, $0.5 \mu 1$ of NSC suspension was injected at depths of $0.25,0.5$ and $0.75 \mathrm{~mm}$, respectively; in 3 of the rats, the NSCs were labeled with BrdU. The rats of the NSC + EPO group underwent NSC transplantation and received an intraperitoneal injection of EPO $(5,000 \mathrm{U} / \mathrm{kg})$ once per day for 7 days; BrdU-labeled NSCs were used in 3 of the rats. The rats in the EPO group received an intraperitoneal injection of EPO $(5,000 \mathrm{U} / \mathrm{kg})$, once per day for 7 days and were transplanted with a gelatin sponge injected with $10 \mu \mathrm{l}$ DMEM/F12 medium. Control group rats received a gelatin sponge injected with $10 \mu \mathrm{l}$ DMEM/F12 medium after modeling and an intraperitoneal injection of normal saline.

Basso, Beattie and Bresnahan (BBB) scoring of rat hindlimb motor function. BBB scoring (11) was used to evaluate the hindlimb motor function of the rats. All rats were subjected to the hindlimb motor function evaluation at $12 \mathrm{~h}$ prior to surgery, then at 3,7, 14, 28, 42 and 56 days following surgery. Two experienced non-laboratory personnel conducted the scoring under randomized, double-blinded conditions, then calculated an average final score of hindlimb motor function for each group.

NF-200 immunohistochemical staining and FITC-conjugated NF-200 fluorescence-labeled spinal cord nerve fibers. 
Experimental animals survived for 8 weeks after surgery. Subsequent to anesthesia, $4 \%$ paraformaldehyde phosphate solution was used to fix the spinal cord tissue by cardiac perfusion (12). Injured spinal cord tissue was incubated in $30 \%$ sucrose overnight at $4^{\circ} \mathrm{C}$. Samples of spinal cord tissue were subjected to conventional gradient dehydration, transparentization and paraffin embedding. The slices were then subjected to immunohistochemical staining with anti-NF-200 antibody (1:200) for $10 \mathrm{~min}$ at $37^{\circ} \mathrm{C}$. A portion of the chilled spinal cord tissue was longitudinally sliced into sections (thickness $30 \mu \mathrm{m}$ ) and one in every three was examined. NF-200 FITC fluorescence staining was subsequently conducted. Frozen sections were dried, then washed three times with $0.01 \mathrm{M}$ potassium-containing phosphate-buffered saline (KPBS), for 10 min per wash. Sections were then immersed in $0.3 \%$ Triton X100 of $0.01 \mathrm{M}$ KPBS for $30 \mathrm{~min}$, then washed three times with $0.01 \mathrm{M}$ KPBS. Sections were immersed in NF-200 FITC (1:400; RS-0046R; Shanghai Yan Jin Biological Science and Technology Co., Ltd.), then rehydrated, clarified, paraffinembedded and observed under a fluorescence microscope (TE2000-S; Nikon Corporation, Tokyo, Japan).

Detection of BrdU-labeled NSCs using fluorescence microscopy. Slices of rat spinal cord tissue transplanted with BrdU-labeled NSCs were subjected to conventional dewaxing, then $0.1 \%$ volume fraction of Triton was used to rupture the cells, which were then washed with PBS and blocked using goat serum. Slices were incubated with a mouse anti-BrdU antibody (1:100) overnight at $4^{\circ} \mathrm{C}$ overnight. PBS replaced primary antibody as a negative control. After washing with PBS, the slices were incubated with FITC-labeled goat anti-mouse fluorescent secondary antibody (1:100; 6925-100; BioVision, Inc., Milpitas, CA, USA). Subsequently, slices were washed with PBS and mounted using a glycerol carbonate buffer solution, and the BrdU-labeled NSCs were then observed using immunoglobulin $\mathrm{G}$ fluorescence and a fluorescence microscope.

Statistical analysis. Results are expressed as the mean \pm standard deviation. One-way analysis of variance was used to conduct statistical tests, and Fisher's least significant difference method to conduct a pairwise comparison. $\mathrm{P}<0.05$ was considered to indicate a statistically significant difference. Statistical analysis was conducted using SPSS software, version 13.0 (SPSS, Inc., Chicago, IL, USA).

\section{Results}

Morphological observation and immunohistochemical identification of NSCs cultured in vitro. Single cells isolated from the hippocampal tissue of embryonic rats appeared circular and consistently sized. The majority of the cells cultured for 2-3 days died, while a limited number of cells entered mitosis. The cells exhibited significant nuclear division, with dividing cells gradually forming clusters. After primary culture for 4-5 days, several to dozens of cells grown in suspension were observed to have formed a spherical cell mass in the nutrient solution. The cell clusters exhibited a reduced volume and marked refraction. The clusters appeared predominantly brownish and had clear boundaries with slightly irregular edges and no evident projections. Therefore, the cells were

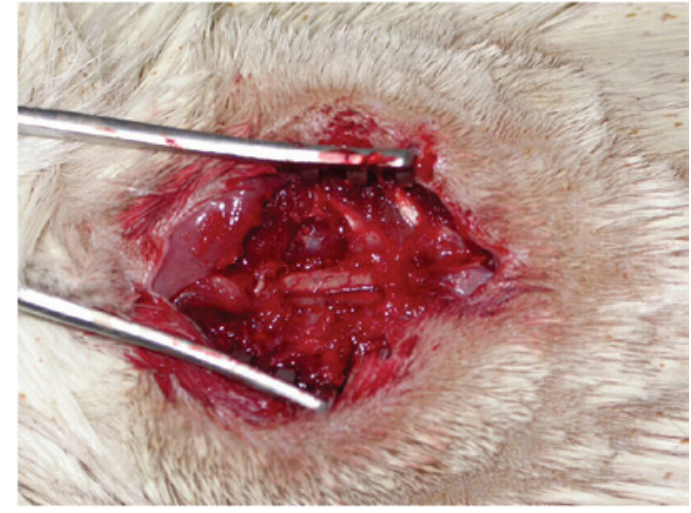

Figure 1. Exposed rat spinal cord.

classed as 'neurospheres'. After 4-5 days of primary culture, the number of neurospheres increased significantly, the center of the cell cluster appeared to have become darker and the coloring at the cluster edge was comparatively light (Fig. 2A). The cells readily adhered to one another, and if not timely passaged, the central cells of the neurosphere typically died due to nutrient deficiency. Neurospheres that had passaged were reprepared into single cell suspension and cultured for 2-3 days. Single cells suspended in the culture solution subsequently divided, forming small clusters of cells. When these cells were cultured for 5-6 days, scattered and varied neurospheres were observed. Larger neurospheres were detected after 7 days, with clear boundaries and strong refraction. These observations indicate that the hippocampal cells were able to self-proliferate. The neural stem cell marker nestin was expressed in the primary cultured and passaged neurospheres. Furthermore, these neurospheres presented with green fluorescence (Fig. 2B), indicating that they were embryo-derived.

Morphological changes in SCI tissue and detection of NSC survival in vivo. In total, 5 rats died during the experiment including 2 control rats and 1 rat each in the EPO, NSC and NSC + EPO groups. The causes of death included bladder rupture, hematuria and bedsores. After 8 weeks, the injured spinal cord tissue darkened in color due to scar tissue formation and was mildly atrophied (Fig. 3). NF-200 staining of the longitudinal slices of injured spinal cord tissue is displayed in Fig. 4. The transected spinal cord tissue of the control and EPO group rats was atrophied, and transverse areas were replaced by transparent, non-colored scar tissue. A large number of holes were observed between the white and gray matter of the spinal cord. The transected spinal cord tissue exhibited mild atrophy in the NSC group, and the disorderly regeneration of nerve fibers was visible in the cross-sectional area, with no continuous nerve fibers traversing the injured region of the spinal cord. The transected spinal cord tissue sections from the NSC + EPO group rats exhibited mild atrophy, with a large quantity of regenerated nerve fibers in a disorderly state in the cross-sectional area, and numerous continuous nerve fibers traversing the injured region. A limited number of holes were detected between the white and gray matter of the spinal cord. In NSCs that succumbed to apoptosis, the BrdU-labeled nucleus exhibited fragmentation and degradation, and was engulfed and 
A

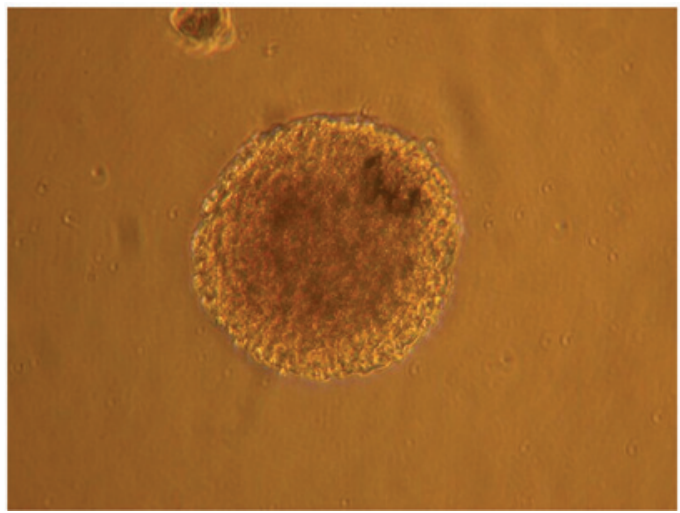

B

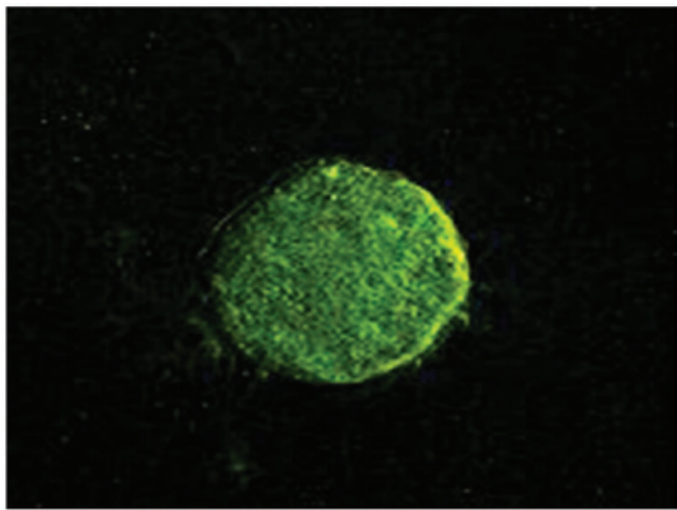

Figure 2. Neural stem cells. (A) Neural stem cell sphere (magnification, x200); (B) neural stem cell glomus visualized using nestin immunofluorescence (fluorescein isothiocyanate; magnification, x200).

A

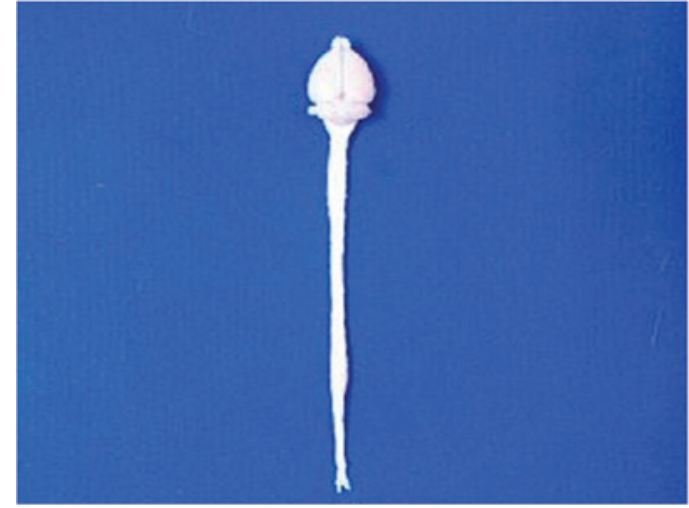

B

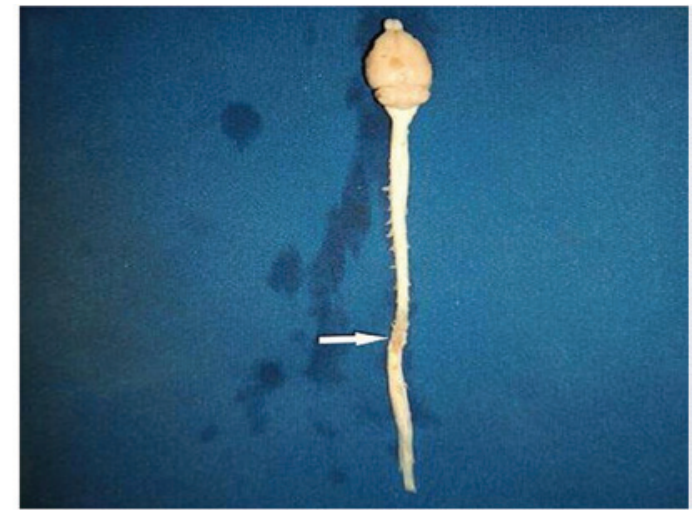

Figure 3. Rat spinal cord. (A) Normal and (B) injured spinal cord.

A

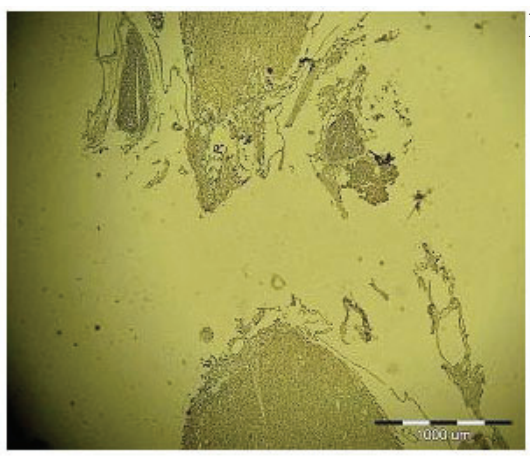

B

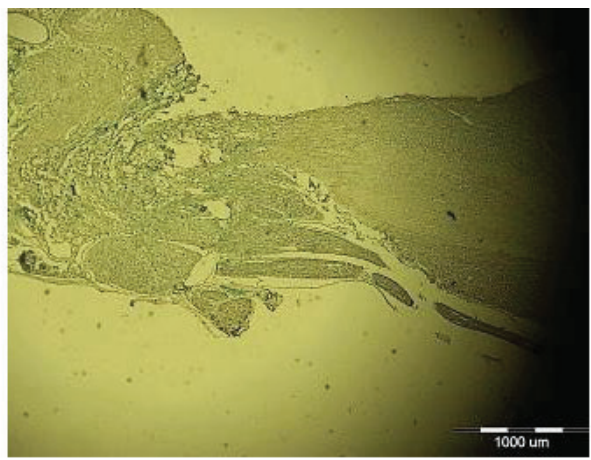

Figure 4. Neurofilament-200 immunohistochemical staining of longitudinal sections at 8 weeks after treatment. (A) Limited axonal regeneration and marked atrophy of the spinal cord stumps were observed in control group sections. (B) Numerous regenerated axones were displayed at the rostral side (left) of spinal cord stumps in the neural stem cell + erythropoietin group, and continuous neurofibers traversed the injured region of the spinal cord.

cleared by macrophages; therefore, these NSCs expressed no orange fluorescence. BrdU-labeled NSCs were observed under a fluorescence microscope (Fig. 5A); numerous labeled NSCs were visible. Some of the stem cells migrated in the directions of the head and tail, demonstrating that a considerable number of NSCs survived after 8 weeks. In the experiment, numerous FITC-conjugated anti-NF-200 antibody-labeled nerve fibers were detected that had regenerated at the side of the transected area closest to the rat's head in the NSC + EPO group, which had regenerated in a disorderly manner and penetrated the tail side of the damaged zone. However, only a small number of regenerated nerve fibers were detected in the control and EPO groups, which did not traverse the damaged zone (Fig. 6).

$B B B$ scoring of rat hindlimb motor function. A normal rat hindlimb BBB score is 21 . Following transective SCI in rats, the $\mathrm{BBB}$ scores of the four groups decreased to 0 within 3 days. The rat hindlimb motor function of the four groups exhibited different degrees of recovery at various time points after the injury. Recovery was most marked in the NSC + EPO and NSC groups, which exhibited recovery of motor function after 1 week, an increased rate of recovery between 2-5 weeks, 

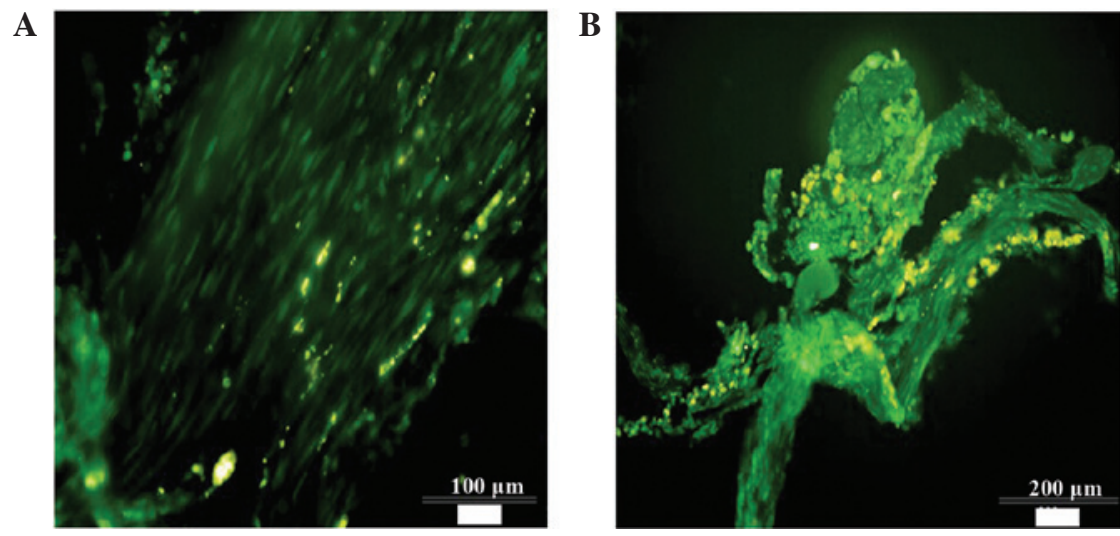

Figure 5. Neural stem cells (NSCs) labeled with Hoechst (saffron yellow) and neurofibers immunofluorescently labeled with fluorescein isothiocyanate neurofilament-200 antibody (green) in the NSC + EPO group at 8 weeks after treatment. (A) NSCs migrated along with regenerated axons and exhibited good survival. (B) NSCs with regenerated axons, guiding and promoting axonal regeneration. EPO, erythropoietin.

A

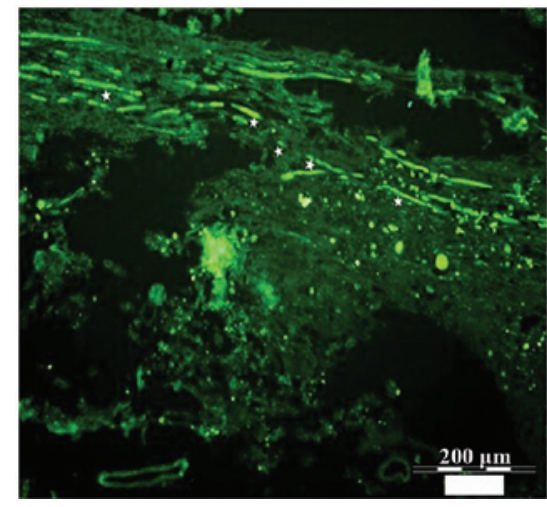

B

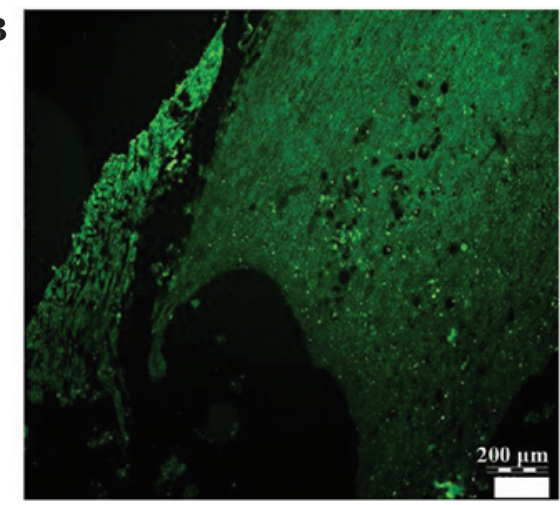

Figure 6. Neurofibers immunofluorescently labeled with fluorescein isothiocyanate neurofilament-200 antibody at 8 weeks after treatment. (A) Limited axonal regeneration was displayed at the rostral side (left) of spinal cord stumps in the NSC + EPO group, and continual neurofibers traversed the injured region of the spinal cord. (B) A few regenerated axons and atrophy of spinal cord stumps were observed in the control group.

and significantly slower recovery between 6-8 weeks (Fig. 7). The results for the EPO, NSC, NSC + EPO and control groups are presented and compared in Table I. At 1-4 weeks after the surgery, the difference between the NSC and EPO groups was significant $(\mathrm{P}>0.05)$. However, after 1 week a statistically significant difference was detected between the NSC and EPO groups $(\mathrm{P}<0.05)$, and the difference between the NSC + EPO and the other groups was statistically significant $(\mathrm{P}<0.05)$. At 8 week after treatment, the fore- and hindlimb motor function of the experimental rats in the NSC + EPO group was coordinated and regular, and the hindfoot was able to bear the rat's body weight, but was not able to lift the body off the ground in the. The motor coordination function of rats in the NSC group was poor, with the hindfeet being able to bear weight in a limited number of rats while the remainder of the rats had hindlimbs with bent toes and poor weight-bearing function. In the control and EPO groups, the rat hindlimb motor function was uncoordinated and exhibited irregular sliding.

\section{Discussion}

In the early 1990s, Reynolds, Tetzlaff and Weiss (13) isolated a cell population from adult rat striatum, which was able to continually proliferate in vitro and possessed multiple differentiation potential. Therefore, the authors proposed the concept

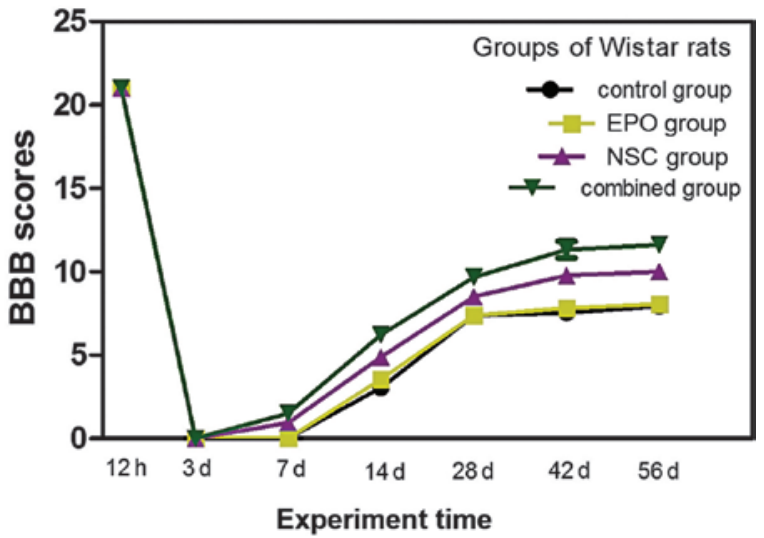

Figure 7. BBB scores of hindlimb movements following spinal cord injury. BBB, Basso, Beattie and Bresnahan; EPO, erythropoietin; NSC, neural stem cell.

of 'NSCs', which exhibit the following characteristics (14): i) The capacity to self-renew, and thus maintain a stable cell population; ii) multi-directional differentiation potential, able to divide into various cell types associated with the nervous system, including neurons, astrocytes and oligodendrocytes; iii) the maintenance of a primitive undifferentiated state, without exhibiting any specific signs of cell maturation. 
Table I. Basso, Beattie and Bresnahan scoring of hindlimb movements following spinal cord injury (mean \pm standard deviation).

\begin{tabular}{lcccc}
\hline Time point & Control & EPO & NSC & NSC + EPO \\
\hline 12 h before & 21.00 & 21.00 & 21.00 & 21.00 \\
7 days after & $0.00 \pm 0.00$ & $0.00 \pm 0.00$ & $0.94 \pm 0.10^{\mathrm{a}}$ & $1.50 \pm 0.26^{\mathrm{a}, \mathrm{b}}$ \\
14 days after & $3.06 \pm 0.36$ & $3.56 \pm 0.32$ & $4.89 \pm 0.60^{\mathrm{a}}$ & $6.22 \pm 0.38^{\mathrm{a}, \mathrm{b}}$ \\
28 days after & $7.38 \pm 0.30$ & $7.39 \pm 0.34$ & $9.50 \pm 0.3^{\mathrm{a}}$ & $9.67 \pm 0.43^{\mathrm{a}, \mathrm{b}}$ \\
42 days after & $7.56 \pm 0.36$ & $7.83 \pm 0.38$ & $10.0 \pm 0.31^{\mathrm{a}}$ & $1.33 \pm 0.50^{\mathrm{a}, \mathrm{b}}$ \\
56 days after & $7.94 \pm 0.35$ & $8.06 \pm 0.31$ & $11.61 \pm 0.46^{\mathrm{a}, \mathrm{b}}$
\end{tabular}

${ }^{\mathrm{a}} \mathrm{P}<0.05$ vs. control and EPO groups; ${ }^{\mathrm{b}} \mathrm{P}<0.05$ vs. NSC group. Repeated measures follow Fisher's least significant difference test. EPO, erythropoietin; NSC, neural stem cells.

Further investigation indicated a potential use for NSCs in the treatment of SCI. NSCs transplanted into a host body were observed to migrate toward the site of SCI, function as chemokines, differentiate into various cell types associated with the nervous system, replace damaged nerve cells and rebuild synaptic connections, all of which may promote the recovery of neurological function. Nakamura et al (15) observed that transplanted NSCs are able to survive, differentiate and migrate in a host body, and effectively promote the recovery of limb motor function. Furthermore, transplanted NSCs exhibit neurotrophic effects and are able to differentiate into neurons and glial cells in the area of SCI. These nerve cells are able to secrete a variety of neurotrophic factors, which improve the local internal environment of an area of SCI, promote the regeneration of axons, repair damaged nerve cells and activate the sequential expression of regeneration-associated genes. Transplanted cells may produce a variety of types of extracellular matrix, filling the cavity produced as a result of SCI and providing support for the regeneration of axons. In addition, transplanted NSCs are able to differentiate into oligodendrocytes, which promote the regeneration of nerve fibers and induce the myelinization of demyelinated nerve fibers (16-18).

Initially, EPO was only known to function as an endocrine hormone in hematopoietic cells. However, studies have indicated that EPO additionally functions as a novel type of organization protection factor, by exerting neurotrophic and neuroprotective effects (19). Possible mechanisms underlying this function of EPO may include its capacity to promote the proliferation and differentiation of NSCs, and the regeneration of nerves. Wang et al (20) used a spinal cord transection injury model to observe the effects of EPO on the proliferation, migration and survival of transplanted BrdU-labeled NSCs in rats with SCI. The results suggested that EPO promotes the survival and migration of NSCs in damaged spinal cord tissue and accelerates the recovery of neural function; ii) EPO is able to mitigate the rate of apoptosis of nerve cells. A previous study revealed (21) that EPO is able to protect neurons from death due to hypoxia and other factors, including excitotoxicity and glucose deprivation in vitro. In addition, EPO is able to immediately prevent the cells of spinal nerve roots from undergoing apoptosis in vivo following crush injury. In a rat model of spinal cord compression, it was observed that EPO exerted a protective effect on nerve cells (22).
In the present study, NSCs extracted from fetal rat hippocampal tissue were cultured in vitro, which significantly increased the number, activity and purity of the primary NSCs. The NSCs exhibited favorable cloning ability and were stably passaged, which facilitated subsequent experiments. In the present study, complete transection of the spinal cord was used to establish the rat model of SCI. Although the rat model does not completely simulate clinical SCI, the complete transection method produces identical injury in each experimental group, thus producing more reliable results. In rats that receive no further treatment following modeling, BBB scores are generally $<10$, with the majority of scores $\sim 8$.

No significant recovery in hindlimb motor function was exhibited by any of the groups within the first 7 days after the induction of SCI. The hindlimb motor function of each group recovered to varying degrees between 7 and 56 days after SCI induction. The hindlimb function recovery in the NSC + EPO group was significantly improved compared with that in the other groups, while the difference between the control and EPO groups was not statistically significant. The rate of hindlimb function recovery of each group was most marked between 7 and 28 days after SCI. The BBB score of the NSC + EPO group was $>10$ for certain rats at 28 days after treatment, indicating that the combined treatment significantly improved the rate of recovery of spinal cord function. The detection of BrdU-labeled NSCs demonstrated that transplanted NSCs were able to survive and migrate in the injured spinal cord tissue. After 8 weeks, injured spinal cord tissue darkened in color due to scar tissue formation, and exhibited mild atrophy. Currently, axonal regeneration is used as a marker of neural regeneration and repair.

NF-200 are a major component of the framework of nerve cell bodies and axons. As a result of the degeneration and necrosis of axons in SCI tissue, the NF-200 that constitutes the framework of nerve axons was reduced and was in certain cases replaced entirely by scar tissue. NF-200 staining of the longitudinal slices of injured spinal cord tissue revealed that the transected spinal cord tissue in the control and EPO groups exhibited atrophy, and transverse areas were replaced by transparent, non-colored scar tissue. Furthermore, a large quantity of hole formation was observed between the white and gray matter of the spinal cord. The spinal cord tissue in the NSC group exhibited mild atrophy, and the disorderly regeneration of nerve fibers was evident in the cross-sectional 
area, with no continuous nerve fibers traversing the area. The transected spinal cord tissue was mildly atrophied in the $\mathrm{NSC}+\mathrm{EPO}$ group, with extensive disorderly regeneration of nerve fibers and numerous continuous nerve fibers traversing the cross-sectional area. Furthermore, a limited degree of hole formation was observed between the white and gray matter of the spinal cord.

In conclusion, the results of the present study suggest that the transplantation of NSCs alone or combined with EPO was able to promote the recovery of hindlimb motor function in a rat model of SCI. These regenerative effects were most evident in the NSC + EPO group. Therefore, the present study indicates that NSC transplantation combined with EPO is an effective intervention for treating SCI, and further studies are required to evaluate the applicability of this treatment to clinical contexts.

\section{Acknowledgements}

This study was supported by a grant from the Inner Mongolia Natural Science Foundation (grant no. 2011MS1139).

\section{References}

1. Wu QL, Li QG and Liu K: Stem cell transplantation for treatment of spinal cord injury. Zhong Guo Zu Zhi Gong Cheng Yan Jiu Yu Lin Chuang Kang Fu 12: 2343-2346, 2008 (In Chinese).

2. Rangarajan V and Juul SE: Erythropoietin: Emerging role of erythropoietin in neonatal neuroprotection. Pediatr Neurol 51: 481-488, 2014

3. Matis GK and Birbilis TA: Erythropoietin in spinal cord injury. Eur Spine J 18: 314-323, 2009.

4. Yuan LL, Du HM, Guan YJ and Kong YH: Differentiation of rat embryonic cerebral cortex neural stem cells cultured by erythropoietin in vitro. Zhong Guo Zu Zhi Gong Cheng Yan Jiu Yu Lin Chuang Kang Fu 15: 9174-9177, 2011 (In Chinese).

5. Liu K, Wang HY, He Y, Zhang YZ and Wang ZC: Experiment on cultivation and identification of neural stem cells in embryonic rats. Zhong Guo Xian Dai Shen Jing Ji Bing Za Zhi 6: 52-56, 2006 (In Chinese).

6. Zhao Z, Hu H and Shi S: The optimization of the method of culturing neural stem cells in neonatal rat brain. Zhongguo Xiu Fu Chong Jian Wai Ke Za Zhi 19: 544-547, 2005 (In Chinese).

7. Zhang ZS, Li JH, Zhang YZ and Wang ZC: Effect of cell density on proliferation and differentiation of human fetal neural stem cells. Shou Du Yi Ke Da Xue Xue 4: 180-183, 2003 (In Chinese).
8. Zhang XR, Guo GH, Liu DW and Peng Y: Separation, culture and BrdU labeling of human bone marrow mesenchymal stem cells. Zhong Guo Zu Zhi Gong Cheng Yan Jiu Yu Lin Chuang Kang Fu 13: 3618-3622, 2009 (In Chinese).

9. Meng BL, Ba YC, Song SN, Chen SS, Li LY and Wang TH: Establishment of spinal cord transection injury models in rats. Zhong Guo Zu Zhi Gong Cheng Yan Jiu Yu Lin Chuang Kang Fu 15: 1215-1218, 2011 (In Chinese).

10. Zhang SX, Huang F, Gates M, White J and Holmberg EG: Extensive scarring induced by chronic intrathecal tubing augmented cord tissue damage and worsened functional recovery after rat spinal cord injury. J Neurosci Methods 191: 201-207, 2010.

11. Basso DM, Beattie MS and Bresnahan JC: Graded histological and locomotor outcomes after spinal cord contusion using the NYU weight-drop device versus transection. Exp Neurol 139: 244-256, 1996.

12. Talac R, Friedman JA, Moore MJ,Lu L, Jabbari E, Windebank AJ, Currier BL and Yaszemski MJ: Animal spinal cord injury for evaluation of tissue engineering treatment strategies. Biomaterials 25: 1505-1510, 2004.

13. Reynolds BA, Tetzlaff $W$ and Weiss S: A multipotent EGF-responsive striatal embryomic progenitor cell produces neurons and astrocytes. J Neurosci 12: 4565-4574, 1992.

14. Taupin P: BrdU immunohistochemistry for studying adult neurogenesis: Paradigms, pitfalls, limitations and validation. Brain Res Rev 53: 198-214, 2007.

15. Nakamura M, Toyama Y and Okano H: Transplantation of neural stem cells for spinal cord injury. Rinsho Shinkeigaku 45: 874-876, 2005 (In Japanese).

16. Nandoe Tewarie RS, Hurtado A, Bartels RH, Grotenhuis A and Oudega M: Stem cell-based therapies for spinal cord injury. J Spinal Cord Med 32: 105-114, 2009.

17. Foret A, Quertainmont R, Botman O, Bouhy D, Amabili P, Brook G, Schoenen J and Franzen R: Stem cells in the adult rat spinal cord: Plasticity after injury and treadmill training exercise. J Neurochem 112: 762-772, 2010.

18. Garbossa D, Boido M, Fontanella M, Fronda C, Ducati A and Vercelli A: Recent therapeutic strategies for spinal cord injury treatment: Possible role of stem cells. Neurosurg Rev 35: 293-311, 2012.

19. Subirós N, Del Barco DG and Coro-Antich RM: Erythropoietin: Still on the neuroprotection road. Ther Adv Neurol Disord 5: 161-173, 2012.

20. Wang ZW, Guan QK, Zhou WK, Zhang XZ and Xu DW: Effect of erythropoietin on survival and migration of neural stem cells transplanted into injured spinal cord of rats. Zhong Guo Zu Zhi Gong Cheng Yan Jiu Yu Lin Chuang Kang Fu 16: 6736-6740, 2012 (In Chinese).

21. Lee KB, Choi JH, Byun K, Chung KH, Ahn JH, Jeong GB, Hwang IK, Kim S, Won MH and Lee B: Recovery of CNS pathway innervating the sciatic nerve following transplantation of human neural stem cells in rat spinal cord injury. Cell Mol Neurobiol 32: 149-157, 2012.

22. Mofidi A, Bader A and Pavlica S: The use of erythropoietin and its derivatives to treat spinal cord injury. Mini Rev Med Chem 11: 763-770, 2011. 